\title{
Unexpected drop of dynamical heterogeneities in colloidal suspensions approaching the jamming transition
}

\author{
PIERRE BALLESTA* ${ }^{*}$ AGNÈS DURI* AND LUCA CIPELLETTI ${ }^{\dagger}$ \\ LCVN UMR 5587, Université Montpellier 2 and CNRS, 34095 Montpellier Cedex 5, France \\ *Present Address: School of Physics, The University of Edinburgh, Edinburgh EH9 3.JZ, UK (P.B.); HASYLAB at DESY, D-22603 Hambourg, Germany (A.D.) \\ †e-mail: lucacip@Icvn.univ-montp2.fr
}

In supercooled molecular fluids or concentrated colloids and grains, the dynamics slow down markedly with no distinct structural changes as the glass ${ }^{1}$ or the jamming ${ }^{2}$ transition is approached. There is now ample evidence that structural relaxation in glassy systems can only occur through correlated rearrangements of particle 'blobs' of size $\xi$ (refs 3-7), leading to dynamics that are heterogeneous both in time and in space. On approaching these transitions, $\xi$ grows in glass-formers ${ }^{6-8}$, colloids ${ }^{3,4,9}$ and driven granular materials ${ }^{10}$ alike, strengthening the analogies between the glass and the jamming transitions and providing a possible explanation for the slowing down of the dynamics. However, little is known yet on the behaviour of dynamical heterogeneity very close to dynamical arrest. Here, we measure in colloids the maximum of a 'dynamical susceptibility', $\chi^{*}$, that quantifies the temporal fluctuations of the dynamics, the growth of which is usually associated with that of $\xi$ (ref. 11). We find that $\chi^{*}$ initially increases with particle volume fraction, but drops markedly very close to jamming. We show that this behaviour results from the competition between the growth of $\xi$ and the reduced particle displacements associated with rearrangements in very dense suspensions, unveiling a richer-than-expected scenario.

Dynamical heterogeneity is a key ingredient in many of the most advanced attempts to understand and rationalize the glass and the jamming transitions. The recent observation of a critical-like growth of temporal and spatial dynamical fluctuations in a two-dimensional athermal system approaching jamming ${ }^{10}$, similar to that hypothesized for glass-formers ${ }^{12}$, has raised hope that the glass and the jamming transitions may be unified, calling at the same time for further, tighter experimental verifications. Here, we investigate temporal dynamical heterogeneity in a three-dimensional thermal system, concentrated colloidal suspensions close to the maximum packing fraction. Temporal and spatial dynamical heterogeneity are usually closely related: the former can be quantified by a 'four-point dynamical susceptibility' $\chi_{4}$ (the variance of a time-resolved correlation function describing the system relaxation), the amplitude of which is proportional to $\xi^{3}$ (refs 11,13,14). Surprisingly, we find that very close to jamming, temporal and spatial dynamical heterogeneity decouple: whereas $\xi$ continuously grows with volume fraction, the amplitude of temporal fluctuations drops sharply close to the maximum packing fraction. These findings challenge current scenarios where the slowing down of the dynamics on approaching jamming is accompanied by enhanced temporal fluctuations of the dynamics.

The dynamics of colloidal hard spheres slows down markedly close to $\varphi=\varphi_{\mathrm{g}} \approx 0.58$; beyond $\varphi_{\mathrm{g}}$, ultraslow relaxations on short length scales are still observed ${ }^{3}$, until dynamics freeze at the maximum (random) packing fraction, $\varphi_{\max }$. We study concentrated suspensions of polyvinyl chloride xenospheres ${ }^{15}$ suspended in dioctyl phthalate (DOP) at volume fractions larger than $\varphi_{\mathrm{g}}$ and close to $\varphi_{\max }$. The particles have a typical radius of $\approx 5 \mu \mathrm{m}$ and 0.33 size polydispersity, to avoid crystallization; they behave as slightly deformable hard spheres. The dynamics are measured by dynamic light scattering in the highly multiple scattering limit (diffusing wave spectroscopy ${ }^{16}$, DWS). This technique allows particle motion to be probed on length scales of the order of a few tens of nanometres (see the Methods section), which match well the restrained motion of our tightly packed suspensions. A CCD (charge-coupled device) detector is used to record the speckle pattern scattered by the sample. The evolution of the speckle images is quantified by the two-time degree of correlation ${ }^{17}$ $c_{\mathrm{I}}(t, \tau)=\left\langle I_{\mathrm{p}}(t) I_{\mathrm{p}}(t+\tau)\right\rangle_{\mathrm{p}} /\left(\left\langle I_{\mathrm{p}}(t)\right\rangle_{\mathrm{p}}\left\langle I_{\mathrm{p}}(t+\tau)\right\rangle_{\mathrm{p}}\right)-1$, where $I_{\mathrm{p}}(t)$ is the scattered intensity at pixel $p$ and time $t$ and $\langle\cdots\rangle_{\mathrm{p}}$ is an average over the CCD pixels.

To follow the evolution of the dynamics, we calculate $g_{2}(t, \tau)-1$, the two-time intensity correlation function obtained by averaging $\mathcal{c}_{\mathrm{I}}(t, \tau)$ over 10-20 CCD frames. Figure 1a shows representative $g_{2}(t, \tau)-1$ functions for all $\varphi$. Owing to the limited acquisition rate of the CCD, the initial decay of $g_{2}(t, \tau)-1$, corresponding to the rattling of particles in the cages formed by their neighbours, is not captured; in the accessible time window, a plateau followed by a final relaxation is observed, indicative of very slow rearrangements. Figure $1 \mathrm{~b}$ shows a typical example of the time dependence of $\tau_{0}$, the characteristic time of the final relaxation obtained by fitting $g_{2}-1$ to a stretched exponential $a(t) \exp \left\{-\left[\tau / \tau_{0}(t)\right]^{\beta(t)}\right\}$. Initially, $\tau_{0}$ grows nearly linearly with $t$, as observed in many glassy systems. However, a stationary regime is eventually reached (the dynamics on length scales larger than those probed here may still slow down, because the sample is unlikely to be fully equilibrated). In this regime, $\tau_{0}$ exhibits surprisingly large fluctuations but no overall increasing trend. A similar behaviour is observed for all volume fractions; all data presented in the following refer 

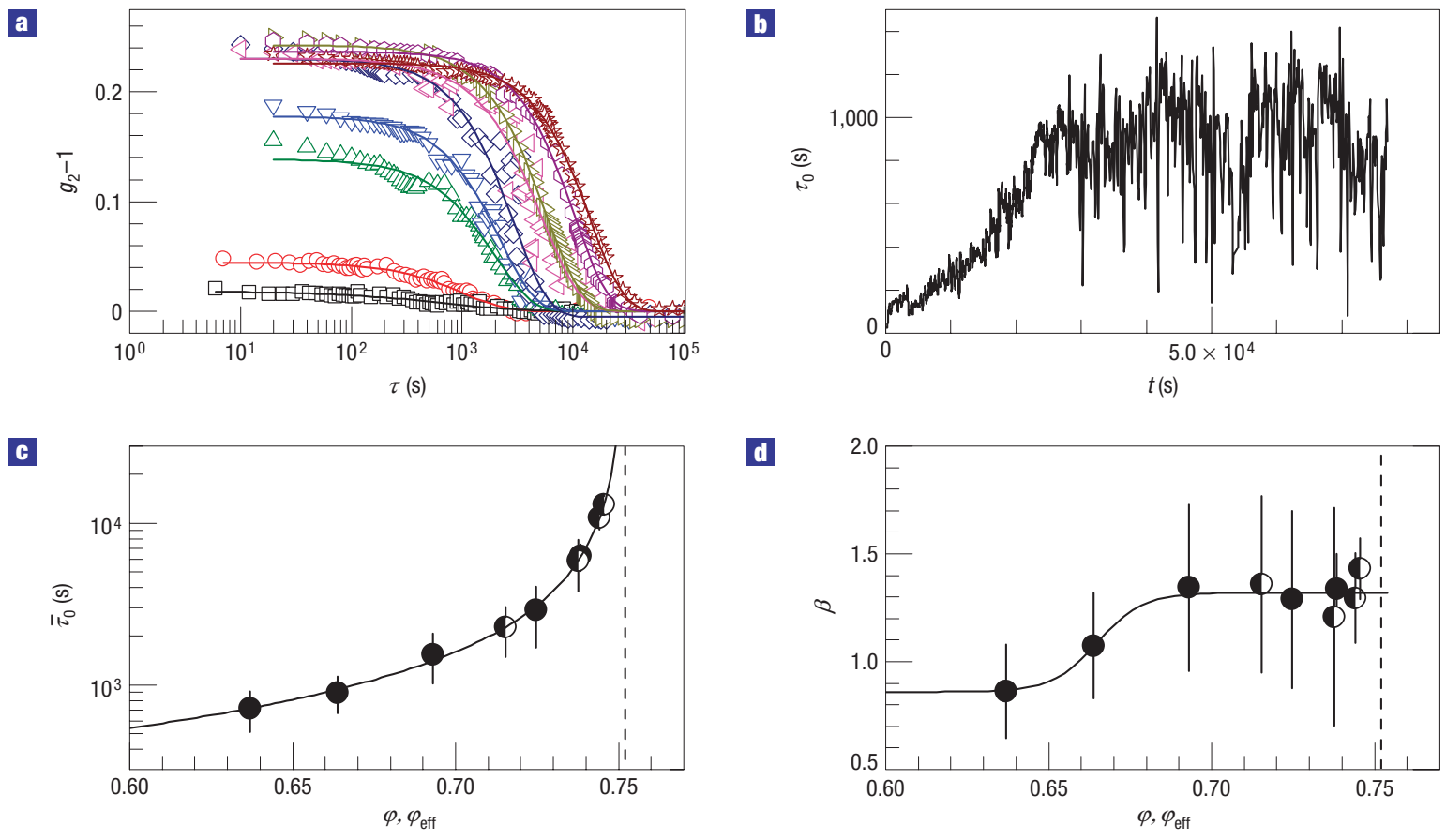

Figure 1 Time and volume fraction dependence of the relaxation of concentrated colloidal suspensions, as probed by time-resolved DWS. a, Symbols: representative two-time intensity correlation functions for all volume fractions investigated. Data are taken in the stationary regime described below and in the text. From bottom to top and left to right, curves refer to samples A-I in Table 1. The lines are stretched exponential fits to the data, $g_{2}(t, \tau)=a(t) \exp \left[-\left(\tau / \tau_{0}(t)\right)^{\beta}(t)\right]$, with $t$ being the time elapsed since loading the cell. For each curve, the fitting parameters $\tau_{0}$ and $\beta$ are close to the average values shown in $\mathbf{c}$ and $\mathbf{d}$. $\mathbf{b}$, Time dependence of $\tau_{0}$, for sample $\mathbf{B}$ $(\varphi=0.664)$. After an initial ageing regime where the dynamics slows down, the system reaches a dynamically heterogeneous stationary state, where $\tau_{0}$ fluctuates significantly without any overall growing trend. c,d, Volume fraction dependence of the relaxation time and the stretching exponent, respectively, averaged over time in the stationary regime. The filled symbols refer to freshly prepared samples; the half-filled circles refer to old samples, the dynamics of which has been re-initialized. The bars are the standard deviations of the distributions over time of $\tau_{0}$ and $\beta$ in the stationary regime. The solid line in $\mathbf{c}$ is a critical-law fit to the growth of $\tau_{0}$ for the fresh samples, yielding a critical exponent $x=1.01 \pm 0.04$ and a critical packing fraction $\varphi_{\max }=0.752$, indicated by the dashed line in $\mathbf{c}$ and $\mathbf{d}$. The solid line in $\mathbf{d}$ is a guide to the eyes.

to the stationary regime, the duration of which is denoted by $t_{\exp }$. We first investigate the $\varphi$-dependence of the average dynamics, as quantified by $\overline{\tau_{0}}$ and $\bar{\beta}$, where $\bar{\cdots}$ denotes a time average over the duration of the stationary regime. As shown in Fig. 1c, $\overline{\tau_{0}}$ continuously increases with $\varphi$. Data taken for freshly prepared samples (filled circles) can be fitted by a critical law, $\overline{\tau_{0}} \sim 1 /\left|\varphi / \varphi_{\max }-1\right|^{x}$, with $\varphi_{\max }=0.752$, consistent with expectations for highly polydisperse samples ${ }^{18}$, and $x=1.01 \pm 0.04$, similarly to ref. 10 . We also study samples that have been aged for several days and the dynamics of which is re-initialized by vigorously stirring and outgassing them (half-filled circles). Their effective volume fraction is higher than the nominal one, owing to the slight swelling of polyvinyl chloride particles suspended in DOP for very long times ${ }^{15}$, allowing them to achieve an even tighter packing. To compare the dynamics of both fresh and aged samples, we assign an effective volume fraction, $\varphi_{\text {eff }}$, to the latter so that their average relaxation time falls on the critical-like curve determined for the fresh samples (Table 1). Figure 1d shows $\bar{\beta}(\varphi)$ : at the lowest volume fraction, the shape of $g_{2}$ is slightly stretched $(\bar{\beta}=0.86<1)$, similarly to what is observed for correlation functions in many glassy systems ${ }^{1}$. Surprisingly, as $\varphi$ increases $\bar{\beta}$ grows above one, finally saturating around 1.3. A similar 'compressed' exponential relaxation has been observed in single ${ }^{19,20}$ and multiple ${ }^{21}$ scattering experiments on systems close to jamming, usually associated with ultraslow ballistic motion.
We quantify the temporal fluctuations of the dynamics by calculating $\chi(\tau, \varphi)$, the relative variance of $c_{\mathrm{I}}$, defined by

$$
\chi(\tau) \equiv \chi(\tau, \varphi)=\overline{\left(c_{\mathrm{I}}(t, \tau)-\overline{c_{\mathrm{I}}(t, \tau)}\right)^{2}} / \bar{a}^{2}
$$

where the $\bar{a}^{-2}$ factor accounts for the $\varphi$-dependence of the amplitude of the final relaxation of $g_{2}-1$; data are furthermore corrected for experimental noise arising from the finite number of probed speckles ${ }^{17}$. The variance introduced above corresponds to the dynamical susceptibility $\chi_{4}$ much studied in simulations of glass-formers ${ }^{11,13}$. Figure $2 \mathrm{a}$ shows both the average correlation function $\overline{g_{2}}-1$ (open circles) and $\chi$ (filled circles) for $\varphi=0.738$. The dynamical susceptibility exhibits a marked peak around $\tau_{0}$, a direct manifestation of dynamical heterogeneity also found in many other glassy systems $s^{5,10,11,13,14,22}$.

Figure $2 b$ shows the height of the peak of the dynamical susceptibility, $\chi^{*}$, as a function of $\varphi$. At the lowest volume fractions, $\chi^{*}$ increases with $\varphi$; using $\varphi_{\max }=0.752$ as obtained from the fit of $\frac{\chi_{0}}{\tau_{0}}(\varphi)$, the data can be fitted by a critical law $\chi^{*} \sim 1 /\left|\varphi / \varphi_{\max }-1\right|^{y}$ with $y=1.5 \pm 0.2$ (line in Fig. $2 \mathrm{~b}$ ), close to $y=1.70$ recently reported for driven grains ${ }^{10}$. This growing trend is also analogous to that observed in simulations of glass-formers ${ }^{6}$ and colloids $s^{3,4,9}$ (albeit at lower $\varphi$ ) and has been interpreted as due to a growing dynamical length scale on approaching dynamical arrest. At higher volume fractions, however, an opposite trend is observed: the amplitude of dynamical fluctuations markedly decreases close 
Table 1 Sample volume fractions and experiment duration. Samples A, B, C, E and $G$ are fresh samples prepared at a volume fraction $\varphi$, for which measurements start right after preparation. Samples D, F, H and I are aged samples initially prepared at a volume fraction $\varphi$, the effective volume fraction of which is slightly larger owing to particle swelling. An effective volume fraction $\varphi_{\text {eff }}$ is assigned to these samples as described in the text. $\overline{\tau_{0}}$ is the average relaxation time of the two-time intensity correlation function $g_{2}(t, \tau)-1$ measured in the regime where the dynamics is stationary. $t_{\exp }$ is the duration of the stationary regime.

\begin{tabular}{lllll}
\hline Sample & $\varphi$ & $\varphi_{\text {eff }}$ & $\overline{\tau_{0}}(\mathrm{~s})$ & $t_{\exp } / \overline{\tau_{0}}$ \\
\hline A & 0.637 & - & 712 & 54.2 \\
B & 0.6638 & - & 897 & 61.8 \\
C & 0.693 & - & 1,547 & 73.7 \\
D & 0.693 & 0.7152 & 2,266 & 55.6 \\
E & 0.7247 & - & 2,889 & 83.8 \\
F & 0.7247 & 0.7377 & 5,862 & 37.3 \\
G & 0.7383 & - & 6,239 & 51.3 \\
H & 0.7383 & 0.7442 & 10,819 & 21.4 \\
I & 0.7383 & 0.7455 & 13,061 & 28.2 \\
\hline
\end{tabular}

to $\varphi_{\max }$. This striking behaviour represents our central result, which challenges current views of dynamical heterogeneity close to dynamical arrest. The unexpected drop of dynamical fluctuations very close to jamming is confirmed by the non-monotonic behaviour of the width of the temporal distributions of $\tau_{0}$ and $\beta$, shown by the vertical bars in Fig. 1c,d. The dispersion of both parameters initially increases with $\varphi$, but eventually is reduced close to $\varphi_{\max }$, further demonstrating reduced dynamical heterogeneity.

We propose that the non-monotonic behaviour of $\chi^{*}$ results from a competition between the growth of $\xi$ on approaching $\varphi_{\max }$ and the reduced particle displacement associated with rearrangement events close to jamming, due to tighter packing ${ }^{23-26}$. Indeed, as $\xi$ increases, fewer statistically independent dynamical regions are contained in the sample, leading to enhanced fluctuations ${ }^{22}$. Conversely, as the particle displacement per event decreases, $\chi^{*}$ is reduced, because more events are required to significantly decorrelate the scattered light and fluctuations on a timescale $\sim \overline{\tau_{0}}$ tend to be averaged out. These competing mechanisms should be quite general and should be observable in a variety of systems, provided that dynamical heterogeneities are probed close enough to dynamical arrest.

We have incorporated these ideas in a simple model for DWS for a dynamically heterogeneous process, significantly extending previous work on the intermittent dynamics of foams ${ }^{27}$ and gels $^{28}$. The dynamics is assumed to be due to discrete, random rearrangement events each affecting a volume $\xi^{3}$; however, in contrast to ref. 27, we assume that several events will be in general necessary to fully decorrelate the phase of scattered photons, because in concentrated suspensions the particle displacement associated with one single event may be much smaller than the wavelength of the light. The two-time field correlation function for a photon crossing the cell along a path of length $s$ may then be written as

$$
g_{1}^{(s)}(t, \tau)=\exp \left[-n_{s}(t, \tau)^{p} \sigma_{\phi}^{2}\right]
$$

(see the Methods section). Here, $n_{s}(t, \tau)$ is the number of events along the path between time $t$ and $t+\tau$ and $\sigma_{\phi}^{2}$ is the variance of the change of phase of a photon due to one single event, proportional to the particle mean squared displacement (MSD) associated with such an event. For a totally uncorrelated change of photon phase due to distinct events, we have $p=1$, whereas in the opposite limit of a perfectly correlated change of phase $p=2$.
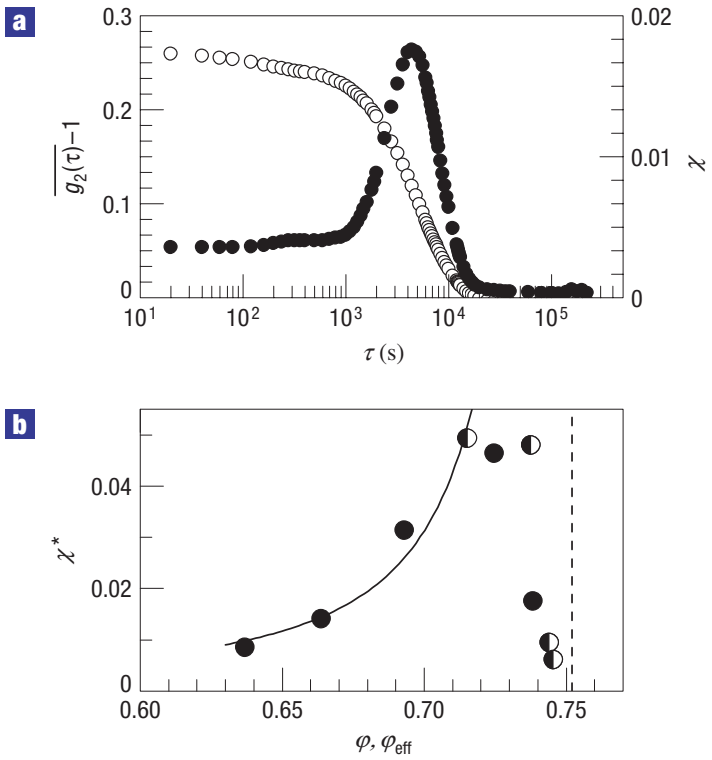

Figure 2 Non-monotonic volume fraction dependence of the dynamical susceptibility close to jamming. a, Average correlation function $\overline{g_{2}(\tau)}-1$ (open symbols and left axis) and dynamical susceptibility (filled symbols and right axis), for sample $G\left(\varphi=0.738\right.$ ). A peak of height $\chi^{*}$ is observed for $\tau \approx \tau_{0}$ (see the Methods section for the normalization of $\chi$ ). $\mathbf{b}$, Volume fraction dependence of $\chi^{*}$ (same symbols as in Fig. 1c,d). The solid line is a critical-law fit to the initial growth of $\chi^{*}$, yielding an exponent $y=1.5 \pm 0.2$. Note the unexpected drop of $\chi^{*}$ near the maximum packing fraction, shown by the dashed line.

We implement our model in Monte Carlo simulations where photon paths are random walks on a square lattice. The lattice sites are affected by random rearrangement events of volume $\xi^{3}$, occurring at a constant rate per unit volume. The simulated degree of correlation is calculated from $c_{I, \text { sim }}(t, \tau)=\left[N_{s}^{-1} \sum g_{1}^{(\mathrm{s})}(t, \tau)\right]^{2}$, where the sum is over $N_{s}=200,000$ photon paths and $g_{1}^{(s)}$ is calculated according to equation (1). The two-time intensity correlation function, $g_{2, \operatorname{sim}}(t, \tau)$, and its fluctuations, $\chi_{\text {sim }}$, are then calculated from $\mathcal{c}_{I, \text { sim }}$ as for the experiments. We vary the control parameters in the simulation, $\xi^{3}, p$ and $\sigma_{\phi}^{2}$, to reproduce the experimental $\varphi$-dependence of $\bar{\beta}$ and $\chi^{*}$. As the particle displacement resulting from one rearrangement - and thus $\sigma_{\phi}^{2}$-is expected to decrease as $\varphi$ grows, owing to tighter particle packing ${ }^{23-26}$, we choose $1 / \sigma_{\phi}^{2}$ as the control parameter against which simulation results are presented, corresponding to increasing volume fractions in Fig. 1b,c. Figure 3 a shows $\bar{\beta}$ versus $1 / \sigma_{\phi}^{2}$. The data are obtained using the values of $\xi^{3}$ shown in Fig. $3 \mathrm{c}$; however, we find that $\bar{\beta}$ depends only very weakly on $\xi^{3}$. For large particle displacements (small $\left.1 / \sigma_{\phi}^{2}\right), \bar{\beta} \lesssim 1$ in fair agreement with the experimental value at the lowest $\varphi$. As particle displacements become increasingly restrained, $\bar{\beta}$ grows and saturates at $\bar{\beta} \approx 1.3$, close to the experimental values at the highest $\varphi$. The saturation value depends on the choice of $p$ : here, $p=1.65$, showing that the change of phase of a photon due to distinct rearrangements is partially correlated. It is unlikely that such a correlation exists for events occurring in non-overlapping regions; in contrast, successive events in the same region will lead to partially correlated changes of phase when the direction of displacement persists during several events. Thus, $p=1.65$ indicates intermittent supradiffusive motion, a behaviour close to the ballistic motion reported for many jammed systems ${ }^{19,20}$. 


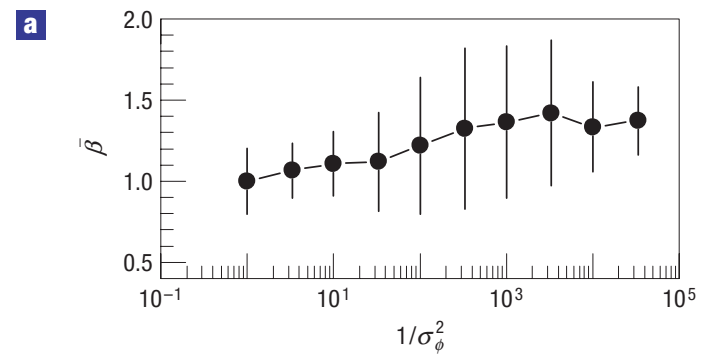

b

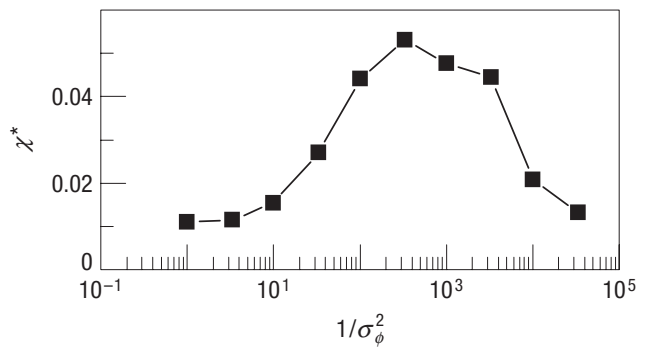

G

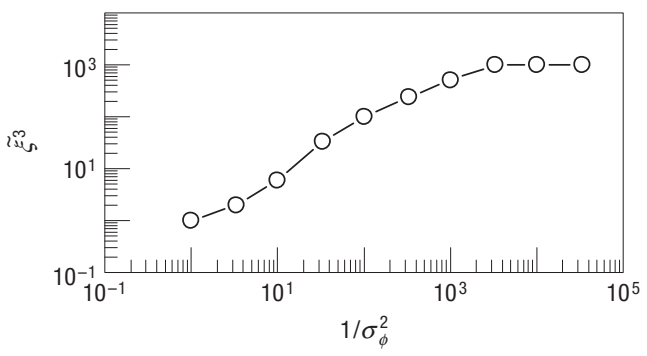

Figure 3 Results of simulations reproducing the non-monotonic behaviour of the dynamical susceptibility observed experimentally. a,b, Time-averaged stretching exponent $\bar{\beta}$ (a) and peak of the dynamical susceptibility $\chi^{*}$ (b) as a function of $1 / \sigma_{\phi}^{2}$, as obtained from the simulations described in the text. The parameter $1 / \sigma_{\phi}^{2}$ on the $x$ axis increases with $\varphi$. In a, the bars indicate the standard deviation of the distribution of stretching exponents. c, The volume $\widetilde{\xi}^{3} \equiv\left(\xi / \ell^{*}\right)^{3}$ of the rearranged regions used as an input in the simulations to reproduce the $\varphi$-dependence of $\bar{\beta}$ and $\chi^{*}$ observed in the experiments. $\xi$ saturates at the value of the smallest dimension of the scattering cell.

Figure $3 \mathrm{~b}$ shows the $1 / \sigma_{\phi}^{2}$-dependence of $\chi^{*}$. The simulations reproduce well both the non-monotonic trend and the absolute values of the experimental $\chi^{*}$. They reproduce also the non-monotonic $\varphi$-dependence of the dispersion of $\beta$ (bars in Fig. 3a), once again matching closely the experimental data. In spite of the drop of dynamical fluctuations close to $\varphi_{\max }, \xi^{3}$ grows steadily with $\varphi$ (Fig. 3c), until rearrangement events extend over the thickness of the cell, corresponding to $\xi \approx 2,000$ particle diameters. System-spanning 'earthquakes' have been reported in simulations of both thermal ${ }^{29}$ and athermal ${ }^{26}$ systems close to dynamical arrest, but have never been observed experimentally.

Our results show that the behaviour of dynamical heterogeneity very close to the jamming transition is much richer and complex than expected. On the one hand, dynamically correlated regions can extend over distances much larger than those previously reported, suggesting that confinement effects, usually observed on the scale of tens of particles at most ${ }^{30}$, may eventually become relevant macroscopically. On the other hand, temporal fluctuations of the dynamics are suppressed, challenging current views of dynamical heterogeneity close to jamming and calling for new theories.
METHODS

\section{EXPERIMENTS}

DWS $^{16}$ experiments are carried out in the transmission geometry, in a cell of thickness $L=2 \mathrm{~mm}$. The photon transport mean path is $\ell^{*} \approx L / 10$. The intensity correlation function $g_{2}-1$ decays to zero on the timescale it takes particles to move on a length scale ${ }^{16} \Lambda \approx \lambda \ell^{*} / L$, with $\lambda$ being the wavelength of the laser in the solvent. Here, $\Lambda \approx 35 \mathrm{~nm}$ for all samples. Note that, as shown, for example, in refs $5,14,28$, the dynamical susceptibility usually depends on the length scale at which the dynamics are probed. It is therefore important to keep this length scale fixed when comparing different samples, as in our experiments. After loading the cell, the samples are vigorously stirred and outgassed to remove any air bubbles. We set $t=0$ at the end of the loading procedure. The particles have a typical radius of $\approx 5 \mu \mathrm{m}$ and a polydispersity of 0.33 (standard deviation of the number distribution of radii normalized by the mean value). The size distribution was obtained in single scattering measurements on very diluted samples, applying the constrained regularization method (CONTIN) to intensity correlation functions measured at scattering angles $\theta=45^{\circ}, 60^{\circ}$ and $90^{\circ}$. We check that in moderately concentrated suspensions $(\varphi=0.28$ and 0.46$)$ the particle MSD measured by DWS increases linearly with time, indicating that on the length scales probed here the particles are brownian. The diffusion coefficient $D=9.02 \times 10^{-16} \mathrm{~m}^{2} \mathrm{~s}^{-1}$ extracted from the MSD using the viscosity of DOP ( $\eta=0.08 \mathrm{~Pa}$ s) is consistent with what is expected from DWS measurements on polydisperse particles of radius $\approx 5 \mu \mathrm{m}$ (ref. 16).

As pointed out in refs 8,17, the experiment duration $t_{\exp }$ should be long enough to fully sample dynamical fluctuations, because $\chi$ would be underestimated if $t_{\exp }$ was shorter than the characteristic time of the fluctuations of the dynamics. By dividing our data into segments of duration $t_{\text {seg }}<t_{\text {exp }}$ and by calculating $\chi$ as a function of $t_{\text {seg }}$, we have checked that for all samples measurements last long enough for $\chi$ to be essentially independent of $t_{\text {exp. }}$. In particular, we find that the relative deviation of the maximum of $\chi$ when processing only half of the available data, $\left|\chi^{*}\left(t_{\exp }\right)-\chi^{*}\left(t_{\exp } / 2\right)\right| / \chi^{*}\left(t_{\exp }\right)$, is on average $\sim 8 \%$ and does not exceed $19 \%$.

In simulations on glass-forming systems, the dynamical susceptibility $\chi_{4}$ is usually normalized by multiplying the variance of the intermediate scattering function-or a similarly defined correlation function—by the number of particles, $N_{\mathrm{p}}$. In our DWS experiments, the number of probed particles is not known precisely, because the scattering volume does not have sharp boundaries (owing to the diffusive nature of the photon paths) and because the particles are polydisperse. In addition, the $N_{\mathrm{p}}$ normalization factor introduced in simulations originates from the fact that in that case the intermediate scattering function is a sum over the contributions of $N_{\mathrm{p}}$ particles, whereas in DWS the field correlation function-and thus $g_{2}-1$ - is expressed as a sum over the contributions of photon paths, rather than particles ${ }^{16}$. We have thus omitted the $N_{\mathrm{p}}$ normalization factor in our definition of the dynamical susceptibility $\chi$, in agreement with previous work ${ }^{17}$. The values of $\chi$ reported here are therefore much smaller than those typically obtained in simulations of dynamically heterogeneous glassy systems.

\section{MONTE CARLO SIMULATIONS}

Photon paths are simulated as random walks on a square lattice with lattice parameter $\ell^{*}$ and dimensions that match those of the experimental cell. In our model of DWS from a temporally intermittent process, the relevant parameter that controls the dynamics is $n_{s}(t, \tau)$, the number of rearrangement events along a path of length $s$ that occurred between times $t$ and $t+\tau$. In analogy with ref. 16 , we thus write $g_{1}^{(s)}=\exp \left[-(1 / 2)\left\langle\Delta \phi^{2}\left(n_{s}\right)\right\rangle\right]$, where $\Delta \phi^{2}\left(n_{s}\right)$ is the change of phase due to $n_{s}$ events along a path of length $s$. By adapting the formalism of ref. 16 to our case, we find $\Delta \phi^{2}\left(n_{s}\right)=(2 / 3) N k_{0}^{2}\left(\ell / \ell^{*}\right)\left\langle\Delta r^{2}\left(n_{s}\right)\right\rangle$, where $N$ is the number of scatterers along the path that have been displaced owing to a rearrangement event and $\left\langle\Delta \phi^{2}\left(n_{s}\right)\right\rangle$ is the MSD of a particle after $n_{s}$ events. $k_{0}$ and $\ell$ are the wave vector of the incident light and the photon scattering mean path, respectively. If the particle displacements due to successive events are totally uncorrelated, $\left\langle\Delta \phi^{2}\left(n_{s}\right)\right\rangle \propto n_{s}$, whereas for perfectly correlated displacements (for example, if the direction of motion persists over successive events) $\left\langle\Delta \phi^{2}\left(n_{s}\right)\right\rangle \propto n_{s}^{2}$. For the sake of generality, we write $\left\langle\Delta \phi^{2}\left(n_{s}\right)\right\rangle=\sigma^{2} n_{s}^{p}$, with $1 \leq p \leq 2$ and where $\sigma^{2}$ is the MSD for one single rearrangement event (except for a numerical factor of order one). The field correlation function then reduces to equation (1). The parameter $p$ governs the shape of the correlation function for $1 / \sigma_{\phi}^{2} \rightarrow \infty$ : 
in this limit, $\overline{g_{1}^{(s)}(\tau)}$ has a compressed exponential shape with a compressing exponent equal to $p$. Summing over all paths with different length $s$ - and thus different decay rates-results in an effective compressing exponent $\bar{\beta}<p$.

Received 21 May 2007; accepted 22 May 2008; published 22 June 2008.

\section{References}

1. Donth, E. The Glass Transition (Springer, Berlin, 2001).

2. Liu, A. J. \& Nagel, S. R. Jamming is not just cool anymore. Nature 396, 21-22 (1998).

3. Weeks, E. R., Crocker, J. C., Levitt, A. C., Schofield, A. \& Weitz, D. A. Three-dimensional direct imaging of structural relaxation near the colloidal glass transition. Science 287, 627-631 (2000).

4. Weeks, E. R., Crocker, J. C. \& Weitz, D. A. Short and long range correlated motion observed in colloidal glasses and liquids. J. Phys. Condens. Matter 19, 205131 (2007).

5. Dauchot, O., Marty, G. \& Biroli, G. Dynamical heterogeneity close to the jamming transition in a sheared granular material. Phys. Rev. Lett. 95, 265701 (2005).

6. Glotzer, S. C. Spatially heterogeneous dynamics in liquids: Insight from simulation. J. Non-Cryst. Solids 274, 342-355 (2000).

7. Ediger, M. D. Spatially heterogeneous dynamics in supercooled liquids. Annu. Rev. Phys. Chem. $\mathbf{5 1}$ 99-128 (2000).

8. Merolle, M., Garrahan, J. P. \& Chandler, D. Space-time thermodynamics of the glass transition. Proc. Natl Acad. Sci. 102, 10837-10840 (2005).

9. Berthier, L. et al. Direct experimental evidence of a growing length scale accompanying the glass transition. Science 310, 1797-1800 (2005).

10. Keys, A. S., Abate, A. R., Glotzer, S. C. \& Durian, D. J. Measurement of growing dynamical length scales and prediction of the jamming transition in a granular material. Nature Phys. 3 260-264 (2007).

11. Lacevičc, N., Starr, F. W, Schroder, T. B., Novikov, V. N. \& Glotzer, S. C. Growing correlation length on cooling below the onset of caging in a simulated glass-forming liquid. Phys. Rev. E 66, 030101 (2002)

12. Biroli, G., Bouchaud, J. P., Miyazaki, K. \& Reichman, D. R. Inhomogeneous mode-coupling theory and growing dynamic length scale in supercooled liquids. Phys. Rev. Lett. 97, 195701 (2006).

13. Franz, S., Donati, C., Parisi, G. \& Glotzer, S. C. On dynamical correlations in supercooled liquids. Phil. Mag. B 79, 1827-1831 (1999).

14. Chandler, D., Garrahan, J. P., Jack, R. L., Maibaum, L. \& Pan, A. C. Lengthscale dependence of dynamic four-point susceptibilities in glass formers. Phys. Rev. E 74, 051501 (2006).

15. Herk, H. H., Bikoles, N. M., Overgerger, C. G. \& Menzes, G. in Encyclopedia of Polymer Science and Engineering (eds Mark, H. E. et al.) (Wiley-Interscience, New York, 2003).

16. Weitz, D. A. \& Pine, D. J. in Dynamic Light Scattering (ed. Brown, W.) 652-720 (Clarendon, Oxford, 1993).

17. Duri, A., Bissig, H., Trappe, V. \& Cipelletti, L. Time-resolved-correlation measurements of temporally heterogeneous dynamics. Phys. Rev. E 72, 051401 (2005).
18. Kansal, A. R., Torquato, S. \& Stillinger, F. H. Computer generation of dense polydisperse sphere packings. J. Chem. Phys. 117, 8212-8218 (2002).

19. Cipelletti, L., Manley, S., Ball, R. C. \& Weitz, D. A. Universal aging features in the restructuring of fractal colloidal gels. Phys. Rev. Lett. 84, 2275-2278 (2000).

20. Bandyopadhyay, R., Liang, D., Harden, J. L. \& Leheny, R. L. Slow dynamics, aging, and glassy rheology in soft and living matter. Solid State Commun. 139, 589-598 (2006).

21. Knaebel, A. et al. Aging behavior of laponite clay particle suspensions. Europhys. Lett. $\mathbf{5 2}$ 73-79 (2000).

22. Mayer, P. et al. Heterogeneous dynamics of coarsening systems. Phys. Rev. Lett. 93, 115701 (2004).

23. Weeks, E. R. \& Weitz, D. A. Properties of cage rearrangements observed near the colloidal glass transition. Phys. Rev. Lett. 89, 095704 (2002).

24. Marty, G. \& Dauchot, O. Subdiffusion and cage effect in a sheared granular material. Phys. Rev. Lett. 94, 015701 (2005).

25. Reis, P. M., Ingale, R. A. \& Shattuck, M. D. Caging dynamics in a granular fluid. Phys. Rev. Lett. 98 188301 (2007).

26. Brito, C. \& Wyart, M. Heterogeneous dynamics, marginal stability and soft modes in hard sphere glasses. J. Stat. Mech.-Theory Exp. L08003 (2007).

27. Durian, D. J., Pine, D. J. \& Weitz, D. A. Multiple light-scattering probes of foam structure and dynamics. Science 252, 686-688 (1991).

28. Duri, A. \& Cipelletti, L. Length scale dependence of dynamical heterogeneity in a colloidal fractal gel. Europhys. Lett. 76, 972-978 (2006).

29. Kob, W. \& Barrat, J. L. Fluctuations, response and aging dynamics in a simple glass-forming liquid out of equilibrium. Eur. Phys. J. B 13, 319-333 (2000).

30. Alcoutlabi, M. \& McKenna, G. B. Effects of confinement on material behaviour at the nanometre size scale. J. Phys. Condens. Matter 17, R461-R524 (2005).

\section{Acknowledgements}

We thank L. Berthier and G. Biroli for illuminating discussions and M. Cloître for providing us with the samples. This work was partially supported by the European MCRTN 'Arrested matter' (MRTN-CT-2003-504712), the NoE 'SoftComp' (NMP3-CT-2004-502235) and ACI JC2076 and CNES grants. L.C. is a junior member of the Institut Universitaire de France, the support of which is gratefully acknowledged.

Author contributions

Experimental work: P.B.; data analysis: P.B., A.D., L.C.; project planning, simulations and writing the paper: L.C.

\section{Author information}

Reprints and permission information is available online at http://npg.nature.com/reprintsandpermissions. Correspondence and requests for materials should be addressed to L.C. 